\title{
Faix, Ann-Christin
}

\author{
Wie verändern sich die subjektiven Theorien von Lehramtsstudierenden \\ über guten inklusiven Unterricht? \\ Grenzen.Gänge.Zwischen.Welten. Kontroversen - Entwicklungen - Perspektiven der \\ Inklusionsforschung. Bad Heilbrunn : Verlag Julius Klinkhardt 2022, S. 246-254
}

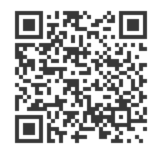

Quellenangabe/ Reference:

Faix, Ann-Christin: Wie verändern sich die subjektiven Theorien von Lehramtsstudierenden über guten inklusiven Unterricht? - In: Grenzen.Gänge.Zwischen.Welten. Kontroversen - Entwicklungen - Perspektiven der Inklusionsforschung. Bad Heilbrunn : Verlag Julius Klinkhardt 2022, S. 246-254 URN: urn:nbn:de:0111-pedocs-238384 - DOI: 10.25656/01:23838

https://nbn-resolving.org/urn:nbn:de:0111-pedocs-238384

https://doi.org/10.25656/01:23838

in Kooperation mit / in cooperation with:

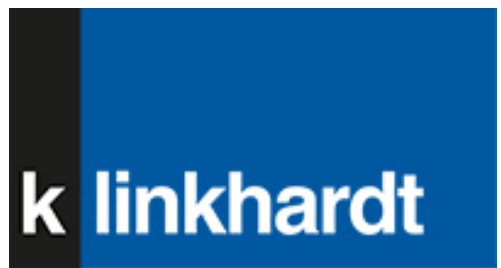

http://www.klinkhardt.de

\section{Nutzungsbedingungen}

Dieses Dokument steht unter folgender Creative Commons-Lizenz: http://creativecommons.org/licenses/by-nc-sa/4.0/deed.de - Sie dürfen das Werk bzw. den Inhalt unter folgenden Bedingungen vervielfältigen, verbreiten und öffentlich zugänglich machen sowie Abwandlungen und Bearbeitungen des Werkes bzw. Inhaltes anfertigen: Sie müssen den Namen des Autors/Rechteinhabers in der von ihm festgelegten Weise nennen. Dieses Werk bzw. der Inhalt darf nicht für kommerzielle Zwecke verwendet werden. Die neu entstandenen Werke bzw. Inhalte dürfen nur unter Verwendung von Lizenzbedingungen weitergegeben werden, die mit denen dieses Lizenzvertrages identisch oder vergleichbar sind.

Mit der Verwendung dieses Dokuments erkennen Sie die Nutzungsbedingungen an.

\section{Terms of use}

This document is published under following Creative Commons-License: http://creativecommons.org/licenses/by-nc-sa/4.0/deed.en - You may copy, distribute and transmit, adapt or exhibit the work in the public and alter, transform or change this work as long as you attribute the work in the manner specified by the author or licensor. You are not allowed to make commercial use of the work. If you alter, transform, or change this work in any way, you may distribute the resulting work only under this or a comparable license.

By using this particular document, you accept the above-stated conditions of use.

\section{Kontakt / Contact:}

\section{peDOCs}

DIPF | Leibniz-Institut für Bildungsforschung und Bildungsinformation

Informationszentrum (IZ) Bildung

E-Mail: pedocs@dipf.de

Internet: www.pedocs.de

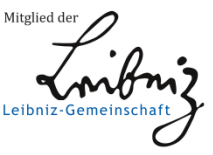




\section{Bernhard Schimek Gertraud Kremsner Michelle Proyer Rainer Grubich Florentine Paudel Regina Grubich-Müller (Hrsg.)}

\section{Grenzen.Gänge. Zwischen. Welten.}

\section{Kontroversen - Entwicklungen - Perspektiven der Inklusionsforschung}


Dieser Titel wurde in das Programm des Verlages mittels eines Peer-Review-Verfahrens aufgenommen. Für weitere Informationen siehe www.klinkhardt.de.

Bibliografische Information der Deutschen Nationalbibliothek Die Deutsche Nationalbibliothek verzeichnet diese Publikation in der Deutschen Nationalbibliografie; detaillierte bibliografische Daten sind im Internet abrufbar über http://dnb.d-nb.de.

2022.hg. () by Julius Klinkhardt.

Coverabbildung: Grafik: (C) Iris Kopera, Foto: kiyopayo/Adobe Stock.

Druck und Bindung: AZ Druck und Datentechnik, Kempten.

Printed in Germany 2022.

Gedruckt auf chlorfrei gebleichtem alterungsbeständigem Papier.

(c) (i)(3)(D) Die Publikation (mit Ausnahme aller Fotos, Grafiken und Abbildungen) ist veröffentlicht unter der Creative Commons-Lizenz: CC BY-NC-SA 4.0 International https://creativecommons.org/licenses/by-nc-sa/4.0/

ISBN 978-3-7815-5924-0 digital doi.org/10.35468/5924

ISBN 978-3-7815-2485-9 print 


\section{Inhalt}

Vorwort der Herausgeber*innen 9

Gertraud Kremsner, Bernhard Schimek und Michelle Proyer

Grenzen.Gänge.Zwischen.Welten.

Kontroversen - Entwicklungen - Perspektiven der Inklusionsforschung

\section{Kontroversen}

Georg Feuser

Die Bühne der Inklusion. Ein Prolog!

oder: Grenzgänge zwischen Welten

Mai-Anh Boger

Risse in der Landschaft der Inklusionsforschung -

Aktuelle Entwicklungen und offene Fragen

Sven Bärmig

Dialektik als Methode

Jan Jochmaring, Lena Bömelburg und Dirk Sponholz

Inklusive Berufsorientierung als Diskurs. Der ,scheinbare' Konsens:

gemeinsame Begriffe - unterschiedliche Ideen

Felix Kappeller

Prothetische Absenz, Articulating Gaps:

Repräsentationskritische Perspektiven auf visuelle Darstellungen

nicht an den Körper gefügter Prothesen

Jana York und Jan Jochmaring

Dilemmata einer inklusiven Arbeitswelt -

Menschen mit Behinderung zwischen Sondersystemen und

Gestaltungschancen einer Arbeitswelt 4.0

Juliane Gerland, Imke Niediek, Julia Hülsken und Marvin Sieger

Kontingenz von Differenzkonstruktionen in der inklusionsorientierten musikalischen Bildung am Beispiel des Umgangs

mit digitalen Musizier-Medien

Pierre-Carl Link

Zur Bedeutung einer befreiungspädagogischen Perspektive für die Inklusions- und Sonderpädagogik 
Katharina Hamisch und Robert Kruschel

Zwischen Individualisierungsversprechen und Vermessungsgefahr -

Die Rolle der Schlüsseltechnologie Künstliche Intelligenz in der inklusiven Schule

Felix Buchhaupt, Dieter Katzenbach, Deborah Lutz und Michael Urban

Zur Kontextualisierung der Inklusionsforschung

Lisa-Katharina Möhlen, Helena Deiß, Seyda Subasi Singh und Michelle Proyer

Lebenswelt(en der) Schule. Internationale Perspektiven zur Inklusion von

Schüler*innen mit Fluchterfahrungen. Eine Perspektive aus der Praxis

Thomas Hoffmann, Cathrin Reisenauer und Hendrik Richter

Helfen als individuelle Erfahrung und soziale Praxis zwischen

Deautonomisierung und Befähigung

\section{Entwicklungen}

Kathrin te Poel

Zur Bedeutsamkeit eigener schüler*innenbiografischer Erfahrungen von angehenden Lehrpersonen für den sich anbahnenden Lehrer*innenhabitus und seine Anerkennungsbezüge

Alina Quante und Oliver Danner

Grenzsetzungen bei Aufgabenbereichen von sonderpädagogischen und

allgemeinen Lehrkräften in inklusiven Settings

Edvina Bešić und Katharina Maitz

Das Boot: Eine Fluchtgeschichte -

Design-Based-Research in der Primarstufe

\section{Brigitte Kottmann}

Der Übergang von der Grundschule zur weiterführenden Schule als

(Soll-)Bruchstelle des Gemeinsamen Lernens

Anne Goldbach und Nico Leonhardt

Elemente von Macht im Kontext einer inklusionssensiblen

Hochschulentwicklung

Michaela Kaiser

Inklusionsbezogene Anforderungswahrnehmung -

Regulativ für (kunstpädagogische) Professionalisierung

Patrick Gollub, Silvia Greiten, Teresa Schkade und Marcel Veber

Schulpraktische Professionalisierung für den Umgang mit Heterogenität ein interdisziplinärer Blick aus hochschuldidaktischen Projekten 
Pierre-Carl Link, Cedric Steinert und Susanne Jurkowski

Implementierung von Inklusion als Querschnittsthema an der

Universität Erfurt durch das Kompetenz- und Entwicklungszentrum

für Inklusion. Inklusionsspezifische Professionalisierung der

Lehrer*innenbildung durch Team-Teaching, Fortbildung und

Online-Lernumgebung

\section{Perspektiven}

Timm Albers, Agnes Filipiak, Katja Franzen und Frank Hellmich

Kompetenzentwicklung im inklusiven Unterricht (KinU) -

eine internationale Perspektive

Katharina Maria Pongratz

Sehnsucht nach Bildung? Über den Einsatz systemischer Fragetechniken in der qualitativen Erwachsenenbildungs-/Weiterbildungsforschung bei Biografieträger*innen mit einer zugeschriebenen geistigen Behinderung

Laura Schwörer, Hannah van Ledden, Pia Algermissen und Mandy Hauser

Zusammenarbeit und Mediennutzung in einer

Partizipativen Forschungsgruppe

David Paulus, Patrick Gollub und Marcel Veber

Forschendes Lernen und Kasuistik. Grenzwelten und Zwischengänge

bezogen auf Reflexivität in der inklusionssensiblen Lehrer*innenbildung

Dietlind Gloystein und Ulrike Barth

Divers denken und handeln! - Theoretische Orientierungen und

Handlungsperspektiven für die Lehrkräftebildung

Ann-Christin Faix

Wie verändern sich die subjektiven Theorien von Lehramtsstudierenden über guten inklusiven Unterricht

Katja Baucke

Internationaler Vergleich als Reflexionsangebot. Eine explorative Studie zur Sicht von Hochschullehrenden auf schulische Inklusion in

Deutschland und Kanada

Bettina Amrhein, Benjamin Badstieber und René Schroeder

Zum Umgang mit als störend wahrgenommenen Handlungsweisen von

Schüler*innen in einem inklusionsorientierten Unterricht - Perspektiven

für die Lehrer*innenbildung (im Förderschwerpunkt emotionale und soziale Entwicklung) 
8 Inhaltsverzeichnis

Andrea Holzinger, Gerda Kernbichler, Silvia Kopp-Sixt, Mathias Krammer und Gonda Pickl

Profilierung für Inklusive Pädagogik (IP) im Lehramt der Primar- und

Sekundarstufe Allgemeinbildung

Timo Finkbeiner und Susanne Eibl

Kooperative Prozesse im technikbezogenen Unterricht 280

Autor*innenverzeichnis 287 


\section{Wie verändern sich die subjektiven Theorien von Lehramtsstudierenden über guten inklusiven Unterricht?}

\section{Einleitung}

Die Realisierung einer inklusiven Bildung erfordert, dass Lehrkräfte „anschlussfähige allgemeinpädagogische und sonderpädagogische Basiskompetenzen für den professionellen Umgang mit Vielfalt in der Schule (...) entwickeln können“ (Hochschulrektorenkonferenz \& Kultusministerkonferenz 2015, 3). Um die Kompetenzentwicklung von Studierenden zu unterstützen, sind neben dem Professionswissen auch handlungsleitende subjektive Theorien ${ }^{1} \mathrm{zu}$ adressieren, da sie die Planung, Wahrnehmung, Durchführung und Reflexion von Unterricht beeinflussen (vgl. Baumert \& Kunter 2006; Wahl 2013). Im Zusammenhang mit inklusivem Unterricht stellen subjektive Theorien eine besondere Herausforderung dar: Die subjektiven Theorien der Studierenden basieren oftmals auf Erfahrungen aus der eigenen Schulzeit (vgl. Pajares 1992). Viele Studierende haben keine Erfahrungen mit inklusivem Unterricht gesammelt, sodass sich ihre subjektiven Theorien möglicherweise als undifferenziert erweisen. Zudem können Prinzipien inklusiven Unterrichts dem eigenen Weltbild und Wertesystem widersprechen, was eine Modifizierung subjektiver Theorien erschweren kann (vgl. Wilde $\&$ Kunter 2016).

\section{Die Bedeutung subjektiver Theorien für professionelles Handeln}

Subjektive Theorien werden definiert als „Kognitionen der Selbst- und Weltsicht, als komplexes Aggregat mit zumindest impliziter Argumentationsstruktur, das auch die zu objektiven (wissenschaftlichen) Theorien parallelen Funktionen der Erklärung, Prognose und Technologie erfüllt" (Groeben, Wahl, Schlee \& Scheele 1988, 19). Im Gegensatz zu Wissen, welches im Fachdiskurs validiert werden und dem Anspruch der Widerspruchsfreiheit genügen muss, ist es für subjektive Theorien hinreichend, dass ihre Träger*innen an ihren Wahrheitsgehalt glauben (vgl.

1 Der Begriff subjektive Theorien wird aufgrund der großen Ähnlichkeit der Konstrukte synonym zu Überzeugungen und Beliefs verwendet (vgl. Reusser \& Pauli 2014). 
Wilde \& Kunter 2016). Da Theoriewissen nur dann handlungsleitend wird, wenn es in subjektive Theorien integriert wird (vgl. Blömeke, Eichler \& Müller 2003), erscheint ein weiter Wissensbegriff sinnvoll, der ,objektives' Wissen als ,wahren“ Teil subjektiver Theorien definiert. Wenn subjektive Theorien handlungsleitend sind, stellt sich die Frage, was ,professionelle' subjektive Theorien kennzeichnet. Professionelle subjektive Theorien weisen eine differenzierte Struktur auf, sind reflektiert und begründet und stehen im Einklang mit wissenschaftlichen Theorien und Erkenntnissen (vgl. Wilde \& Kunter 2016). Dies erfordert, dass subjektive Theorien, die im Widerspruch zu wissenschaftlichen Theorien stehen, bearbeitet werden müssen. Eine weitere Herausforderung besteht darin, dass sich das Handeln von Lehrkräften in widersprüchlichen Handlungsanforderungen - Antinomien - vollzieht, die gleichermaßen Gültigkeit beanspruchen und sich daher nicht auflösen lassen (vgl. Helsper 2002). Professionalität besteht in dieser Perspektive darin, die Widersprüche aushalten und reflektieren zu können (vgl. ebd.). Diese widersprüchlichen Handlungsanforderungen können als kognitiv repräsentierte Konzepte in subjektive Theorien einfließen.

\section{Forschungsinteresse}

Diese Studie hat zum Ziel, die inhaltlichen Veränderungen subjektiver Theorien von Lehramtsstudierenden im Verlauf eines Seminars längsschnittlich zu untersuchen. Dabei ist von Interesse, welche inhaltlichen Aspekte inklusiven Unterrichts durch die Intervention neu hinzutreten oder sich ausdifferenzieren. Auf diese Weise soll überprüft werden, ob die Studierenden konkretere und differenziertere subjektive Theorien über guten inklusiven Unterricht entwickeln. Um die Veränderungen der subjektiven Theorien möglichst offen zu erfassen, wurden Strukturlegepläne ausgewählt, die zugleich als Reflexionsanlass dienten und damit zusätzlich eine hochschuldidaktische Funktion erfüllten. Auf dieser Grundlage sollen Konsequenzen für die Weiterentwicklung des Seminarkonzepts gezogen werden, um die Professionalisierung der Studierenden noch gezielter zu unterstützen.

\section{Methodik}

\subsection{Seminarbeschreibung}

Die Daten wurden im Rahmen des Seminars Ist das guter inklusiver Unterricht? erhoben. Das Seminar wurde in zwei Wintersemestern von jeweils einer Hochschullehrerin der Psychologie und der Erziehungswissenschaft im Teamteaching durchgeführt. Ziel war es, Studierende u.a. durch die Auseinandersetzung mit 
ihren subjektiven Theorien für inklusiven Unterricht zu professionalisieren. ${ }^{2}$ Dazu arbeiteten die Studierenden zunächst ihre subjektiven Theorien über guten inklusiven Unterricht mithilfe einer Strukturlegetechnik heraus, die dann mit wissenschaftlichen Theorien aus den Perspektiven der Pädagogischen Psychologie, der Schulpädagogik und der Inklusiven Pädagogik konfrontiert wurden. Auf dieser Basis haben die Studierenden ein Raster für die Beobachtung inklusiven Unterrichts erarbeitet und an Unterrichtsvideos erprobt. Das Raster kam schließlich in Hospitationen in inklusiven, Good Practice'-Schulen zum Einsatz. Die Hospitationserfahrungen wurden in einer abschließenden Sitzung reflektiert.

\subsection{Sample}

Für die vorliegende Studie wurden die Strukturlegepläne von insgesamt 55 Studierenden analysiert. Davon haben 42 Studierende am ersten und 13 Studierende am zweiten Seminardurchlauf teilgenommen. 35 Studierende waren weiblich, 19 männlich, eine weitere Person ordnete sich keinem Geschlecht zu. 32 Studierende strebten das Lehramt für Gymnasien und Gesamtschulen, 20 das Lehramt für Haupt-, Real-, Sekundar- und Gesamtschulen an, eine Person studierte ein anderes Fach als Lehramt und zwei weitere Personen machten keine Angaben zu ihrem Studiengang. Die Studierenden waren im ersten bis neunten Fachsemester $(M=4,87, S D=1,41)$.

\subsection{Datenerhebung}

Zur Erfassung ihrer subjektiven Theorien haben die Studierenden vor und nach dem Seminar jeweils einen Strukturlegeplan zu gutem inklusivem Unterricht erstellt. Strukturlegepläne sollen subjektive Theorien möglichst adäquat abbilden, indem Inhalte durch Begriffe auf Klebezetteln und Beziehungen durch beschriftete Pfeile repräsentiert werden. Dazu wurden in einem ersten Schritt Begriffe gesammelt und auf den Klebezetteln notiert. In einem zweiten Schritt haben die Studierenden die Zettel auf einer Unterlage sortiert und in eine für sie sinnvolle Struktur gebracht. Die Beziehungen zwischen den Begriffen wurden durch Pfeile kenntlich gemacht.

Um die Art des Zusammenhangs näher zu beschreiben, haben sie die Pfeile mit Relationen beschriftet, die dem Inventar der alltagssprachlichen Flexibilisierungsvariante für Strukturlegetechniken (vgl. Scheele, Groeben \& Christmann 1992) entnommen wurden. Dabei wurden Relationen verschiedener Strukturlegetechniken in Alltagssprache ,übersetzt', sodass sie leichter zugänglich sein sollen (z.B. ,das

2 Für eine ausführliche Beschreibung des Seminarkonzepts: vgl. Faix, Lütje-Klose, Textor \& Wild 2019. Bi ${ }^{\text {professional }}$ wird im Rahmen der gemeinsamen Qualitätsoffensive Lehrerbildung von Bund und Ländern aus Mitteln des Bundesministeriums für Bildung und Forschung gefördert (Förderkennzeichen 01JA1908). 
heißt', ,und', ,erkennbar an', ,notwendige Voraussetzung', ,führt zu'). Bei Bedarf konnten die Studierenden auch eigene Relationen hinzufügen.

Die Arbeitsschritte des Sammelns und Sortierens wechselten sich stetig ab, bis es zu einer Sättigung kam. Am Ende des Seminars haben die Studierenden ihren ursprünglichen Strukturlegeplan überarbeitet, indem sie neue Begriffe auf andersfarbigen Klebezetteln ergänzt haben. Den Studierenden war es freigestellt, die ursprüngliche Struktur beizubehalten und neue Begriffe zu ergänzen oder eine neue Struktur zu wählen (vgl. Faix, Lütje-Klose, Textor \& Wild 2020). Zudem konnten alte Klebezettel vom Strukturlegeplan entfernt und nicht weiter verwendet werden. Aus forschungspraktischen Gründen war es nicht möglich, alle Strukturlegepläne im Dialog-Konsens-Verfahren zu validieren.

\subsection{Datenauswertung}

Die inhaltliche Auswertung der Strukturlegepläne erfolgte in Anlehnung an die zusammenfassende qualitative Inhaltsanalyse nach Mayring (2010). Ziel der Analyse war es, das Material zusammenzufassen und zu reduzieren, um zu zentralen Kategorien zu gelangen. Dazu wurden am Material induktiv Kategorien gebildet. Als Analyseeinheit wurde ein Klebezettel festgelegt, auf welchem meistens nur ein Begriff notiert wurde. Angrenzende Relationen und Klebezettel wurden als Kontexteinheiten in die Analyse einbezogen. Eine Reduktion des Materials erfolgte überwiegend durch die Streichung bedeutungsgleicher Inhalte. Die induktiv am Material gebildeten Kategorien wurden schließlich zu Oberkategorien gruppiert. Das so generierte Kategoriensystem wurde an einem Teil des Materials entwickelt und schließlich am gesamten Material überprüft. Durch die Ermittlung der Kategorienhäufigkeiten wurde das Kategoriensystem quantitativ weiterverarbeitet. Die Anzahl der Kodierungen zu den beiden Erhebungszeitpunkten erlaubt Rückschlüsse im Hinblick auf die Lernentwicklung der Studierenden, da ersichtlich wird, welche Kategorien sich ausdifferenzieren oder neu hinzutreten.

\section{Ergebnisse}

\subsection{Hauptkategorien}

Auf Basis der qualitativen Inhaltsanalyse wurden die fünf Hauptkategorien Inklusionsverständnis, Professionalität der Lehrkraft, Unterricht, Rahmenbedingungen und Ressourcen und (Multiprofessionelle) Kooperation gebildet. Die Kategorie Inklusionsverständnis umfasst Grundorientierungen und Werthaltungen sowie Dimensionen von Heterogenität. Unter der Kategorie Professionalität der Lehrkraft werden mentale Dispositionen (z.B. Wissen, Einstellungen), Persönlichkeitsmerkmale (z.B. Geduld, Konfliktfähigkeit) und Aus-, Fort- und Weiterbildung als Gelegenheiten 
der Professionalisierung für inklusiven Unterricht zusammengefasst. Die Kategorie Rahmenbedingungen und Ressourcen umfasst Rahmenbedingungen (z.B. Lehrpläne, Schulsystem) und Ressourcen (z.B. Geld, Material, Ausstattung). Da beide nicht immer voneinander abgegrenzt werden können, werden sie zu einer Kategorie zusammengefasst. Die Kategorie (Multiprofessionelle) Kooperation umfasst die Zusammenarbeit verschiedener Akteur*innen wie z.B. Sonderpädagog*innen, Schulbegleiter*innen und Eltern. Unter der Kategorie Unterricht werden Dimensionen der konkreten Gestaltung inklusiven Unterrichts zusammengefasst.

Insgesamt ist ein erheblicher Zuwachs der Kodierungen über alle Kategorien hinweg erkennbar. Die quantitativ größten Zuwächse finden sich in der Hauptkategorie Unterricht, deren Kodierungen sich gegenüber dem ersten Erhebungszeitpunkt mehr als verdoppeln. Auch die Anzahl der Kodierungen in den Kategorien Professionalität der Lehrkraft und (Multiprofessionelle) Kooperation verdoppelt sich gegenüber dem ersten Erhebungszeitpunkt nahezu, wobei beide Kategorien deutlich weniger Kodierungen als die Kategorie Unterricht aufweisen. Die Kategorie Inklusionsverständnis weist zu beiden Erhebungszeitpunkten die meisten Kodierungen nach der Kategorie Unterricht auf, wobei die Anzahl der Kodierungen im Vergleich zum ersten Erhebungszeitpunkt nur wenig ansteigt. Die wenigsten Kodierungen weist zu beiden Zeitpunkten die Kategorie Rahmenbedingungen und Ressourcen auf.

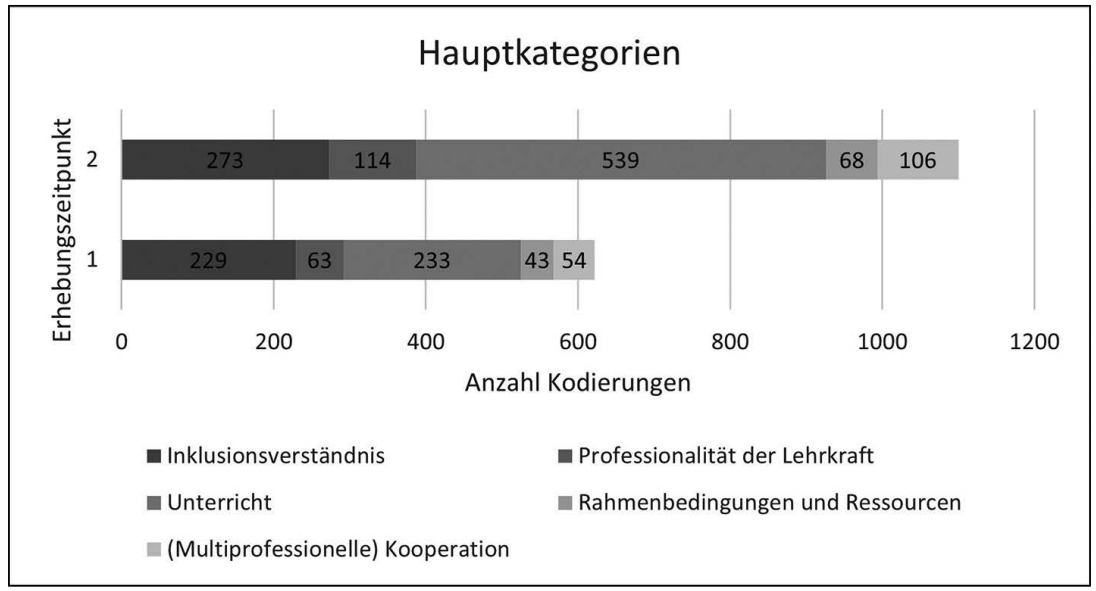

Abb. 1: Kodierungshäufigkeiten der Hauptkategorien zum ersten und zweiten Erhebungszeitpunkt

\subsection{Unterricht}

Da die Hauptkategorie Unterricht für die Fragestellung dieser Studie zentral ist, wird sie im Folgenden gesondert betrachtet. Hier konnten die Unterkategorien 
pädagogisch-psychologische Dimensionen, Differenzierung und Förderung, Herstellung von Gemeinsamkeit, alternative Unterrichtsformen und Klassenklima herausgearbeitet werden. Unter der Kategorie pädagogisch-psychologische Dimensionen wurden Dimensionen zusammengefasst, die von der pädagogisch-psychologischen Forschung als lernwirksam identifiziert wurden (z.B. Klassenführung, kognitive Aktivierung). Die Kategorie Differenzierung und Förderung umfasst differenzierende, individualisierende Maßnahmen, die auch auf den Strukturlegeplänen häufig mit Förderung und Unterstützung verbunden werden. Die Kategorie Herstellung von Gemeinsamkeit umfasst sozialintegrative Maßnahmen, die neben akademischer auch soziale Partizipation ermöglichen sollen (z.B. kooperatives Lernen, gemeinsamer Unterrichtsgegenstand). Unter der Kategorie alternative Unterrichtsformen werden Unterrichtsformen zusammengefasst, die von einem ,traditionellen' Frontalunterricht abweichen (z.B. Wochenpläne, Projektunterricht, offener Unterricht). Da die Kategorie Klassenklima teilweise schwer von Grundorientierungen und Werthaltungen im Unterricht abgegrenzt werden kann, werden hier Aspekte der Lernatmosphäre und des sozialen Miteinanders erfasst (z.B. kein Zeitdruck, Beziehungsarbeit). Zur besseren Übersicht wurden alle Kategorien, die zum zweiten Erhebungszeitpunkt weniger als 40 Kodierungen aufwiesen, unter Sonstige zusammengefasst (z.B. Methoden, Medien und Sozialformen, (Förder-)Diagnostik). Die größten Zuwächse finden sich in der Kategorie pädagogisch-psychologische Dimensionen, die sich gegenüber dem ersten Erhebungszeitpunkt mehr als versechsfacht. Zugleich wird diese Kategorie zum zweiten Erhebungszeitpunkt am häufigsten kodiert. Ein deutlicher Zuwachs findet sich auch für die Kategorie Differenzierung und Förderung, welche zum zweiten Erhebungszeitpunkt am zweithäufigsten kodiert wird. Des Weiteren finden sich Zuwächse für die Kategorien Herstellung von Gemeinsamkeit, alternative Unterrichtsformen und Klassenklima, wobei diese zu beiden Erhebungszeitpunkten deutlich weniger häufig kodiert werden als pädagogisch-psychologische Dimensionen und Differenzierung und Förderung. 


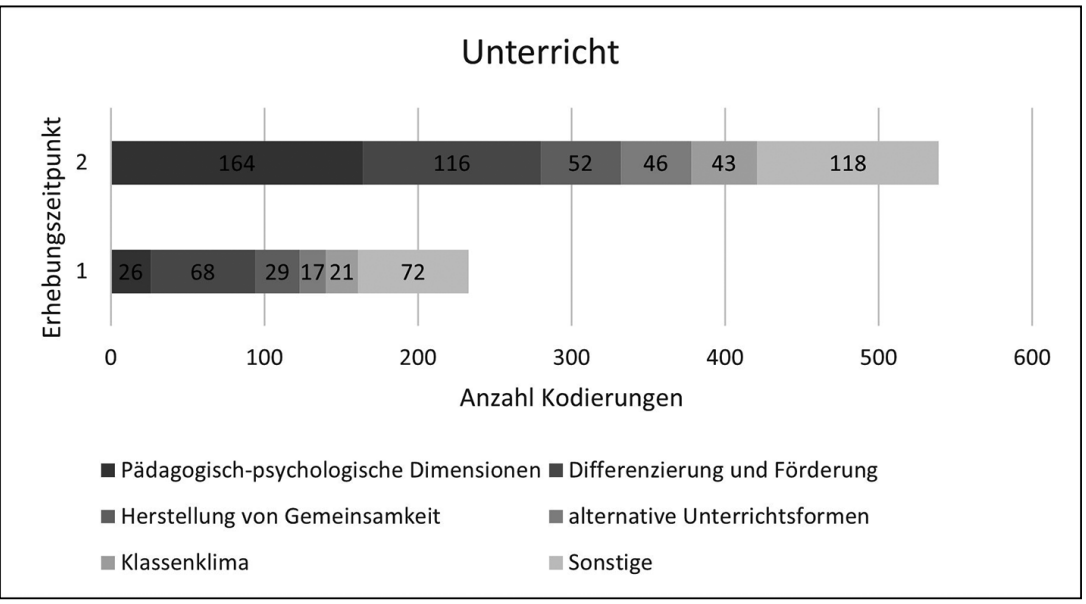

Abb. 2: Kodierungshäufigkeiten innerhalb der Kategorie Unterricht zum ersten und zweiten Erhebungszeitpunkt

\section{Diskussion}

Die Ergebnisse deuten darauf hin, dass die Studierenden zu Beginn der Lehrveranstaltung noch über unspezifische subjektive Theorien verfügten, die sich im Verlauf der Seminare deutlich ausdifferenzierten. Dies zeigt sich an einem deutlichen Zuwachs der Kategorie Unterricht. Die Studierenden verfügen nach den Seminaren über konkretere Vorstellungen, welche Dimensionen für die Gestaltung guten inklusiven Unterrichts relevant sind. Dieses Ergebnis steht im Einklang mit den Zielen des Seminars. Innerhalb der Kategorie Unterricht sind die stärksten Zuwächse in der Kategorie pädagogisch-psychologische Dimensionen zu verzeichnen. Diese enorme Steigerung lässt sich darauf zurückführen, dass psychologische Inhalte im Lehramtsstudium an der Universität Bielefeld vergleichsweise wenig verankert sind, sodass der Lernzuwachs für die Studierenden in diesem Bereich besonders hoch ist. Auffällig ist zu beiden Zeitpunkten das quantitativ ungleiche Verhältnis der Kategorien Differenzierung und Förderung und Herstellung von Gemeinsamkeit. Diesem Befund sollte mit Blick auf die Professionalisierung der Studierenden Aufmerksamkeit geschenkt werden: Differenzierung und die Herstellung von Gemeinsamkeit stehen in einem Spannungsverhältnis (vgl. Werning \& Lütje-Klose 2016). Für inklusiven Unterricht ist die individuelle Förderung von Schüler*innen allein nicht hinreichend, da diese zu Isolation und Stigmatisierung führen kann (vgl. Lütje-Klose 2018). Aus diesem Grund bedarf es einer aktiven und bewussten Herstellung von Gemeinsamkeit, um nicht nur akademische, 
sondern auch soziale Teilhabe zu ermöglichen. Um die Professionalisierung der Studierenden gezielt zu unterstützen, muss ein Bewusstsein für das Spannungsfeld geschaffen werden, um es reflektierend bearbeiten zu können (vgl. Helsper 2002). Gleiches gilt für weitere Spannungsfelder wie z.B. Anerkennung von Differenz und Dekonstruktion von Differenz, die sich auf Ebene untergeordneter Kategorien finden und ebenfalls unterschiedlich häufig benannt werden. Dabei ist zu berücksichtigen, dass das quantitative Verhältnis der Kategorien allein noch nicht aussagt, ob sich die Studierenden dieser Spannungsfelder bewusst sind und ob sie diese reflektieren. Allerdings zeigt sich, dass nur wenige Studierende auf ihren Strukturlegeplänen Relationen verwenden, die ein Spannungsverhältnis anzeigen oder die Spannungsfelder anderweitig kennzeichnen (vgl. Faix 2020). Grundsätzlich gilt, dass die Ergebnisse nicht unabhängig von den Seminarinhalten betrachtet werden können und sich inhaltliche Schwerpunktsetzungen in den Strukturlegeplänen widerspiegeln. Auf dieser Grundlage sollten die Spannungsfelder in zukünftigen Seminaren gezielt thematisiert werden, um die Studierenden dafür zu sensibilisieren und ihre Professionalisierung für inklusiven Unterricht weiter zu unterstützen.

\section{Literatur}

Baumert, J. \& Kunter, M. (2006): Stichwort: Professionelle Kompetenz von Lehrkräften. In: Zeitschrift für Erziehungswissenschaft, 9, 469-520.

Blömeke, S., Eichler, D. \& Müller, C. (2003): Rekonstruktion kognitiver Strukturen von Lehrpersonen als Herausforderung für die empirische Unterrichtsforschung: Theoretische und methodologische Überlegungen zu Chancen und Grenzen von Videostudien. In: Unterrichtswissenschaft, 31, 103-121.

Faix, A.-C. (2020): „Lehrersein ist ja irgendwie von Widersprüchen irgendwo gekennzeichnet«. Eine längsschnittliche Untersuchung Subjektiver Theorien zu gutem inklusivem Unterricht. In: Pädagogische Horizonte, 4, 57-79.

Faix, A.-C., Lütje-Klose, B., Textor, A. \& Wild, E. (2019): Ist das guter inklusiver Unterricht? Mit Videoanalysen und Hospitationen von der Theorie zur Praxisreflexion. In: Herausforderung Lehrer_innenbildung, 2, 1-19.

Faix, A.-C., Lütje-Klose, B., Textor, A. \& Wild, E. (2020): Strukturlegepläne als hochschuldidaktisches Instrument zur Lehrevaluation und Reflexion Subjektiver Theorien. In: Herausforderung Lehrer_innenbildung, 3, 523-537.

Groeben, N., Wahl, D., Schlee, J. \& Scheele, B. (1988): Das Forschungsprogramm Subjektive Theorien: eine Einführung in die Psychologie des reflexiven Subjekts. Tübingen.

Helsper, W. (2002): Lehrerprofessionalität als antinomische Handlungsstruktur. In: Kraul, M., Marotzki, W. \& Schweppe, C. (Hrsg.): Biographie und Profession. Bad Heilbrunn, 64-102.

Hochschulrektorenkonferenz \& Kultusministerkonferenz (2015): Lehrerbildung für eine Schule der Vielfalt. Gemeinsame Empfehlung von Hochschulrektorenkonferenz und Kultusministerkonferenz. Online unter: https://www.kmk.org/fileadmin/Dateien/veroeffentlichungen_beschluesse/2015/2015_03_12-Schule-der-Vielfalt.pdf (letzter Zugriff: 29.05.2019).

Lütje-Klose, B. (2018): Konzeptualisierung von Inklusion und Sonderpädagogik. In: Lütje-Klose, B., Riecke-Baulecke, T. \& Werning, R. (Hrsg.): Basiswissen Lehrerbildung: Inklusion in Schule und Unterricht. Grundlagen in der Sonderpädagogik. Seelze, 9-28. 


\section{Ann-Christin Faix}

Mayring, P. (2010): Qualitative Inhaltsanalyse. In: Mey, G. \& Mruck, K. (Hrsg.): Handbuch Qualitative Forschung in der Psychologie. Wiesbaden, 601-613.

Pajares, M. F. (1992): Teacher's Beliefs and Educational Research: Cleaning Up a Messy Construct. In: Review of Educational Research, 62, 307-332.

Reusser, K. \& Pauli, C. (2014): Berufsbezogene Überzeugungen von Lehrerinnen und Lehrern. In: Terhart, E., Bennewitz, H. \& Rothland, M. (Hrsg.): Handbuch der Forschung zum Lehrerberuf. Münster, 642-661.

Scheele, B., Groeben, N. \& Christmann, U. (1992): Ein alltagssprachliches Struktur-Lege-Spiel als Flexibilisierungsversion der Dialog-Konsens-Methodik. In: Scheele, B. (Hrsg.): Struktur-LegeVerfahren als Dialog-Konsens-Methodik. Ein Zwischenfazit zur Forschungsentwicklung bei der rekonstruktiven Erhebung Subjektiver Theorien. Münster, 152-195.

Wahl, D. (2013): Lernumgebungen erfolgreich gestalten. Vom trägen Wissen zum kompetenten Handeln. Bad Heilbrunn.

Werning, R. \& Lütje-Klose, B. (2016): Einführung in die Pädagogik bei Lernbeeinträchtigungen. München/Basel.

Wilde, A. \& Kunter, M. (2016): Überzeugungen von Lehrerinnen und Lehrern. In: Rothland, M. (Hrsg.): Beruf Lehrer/Lehrerin. Ein Studienbuch. Münster, 299-315. 